\title{
Spatio-temporal channel characterization: theoretical framework and applications in MIMO system design
}

\author{
Viet-Ha Pham • Jean-Yves Chouinard • \\ Dominic Grenier • Huu-Tue Huynh • \\ Xianbin Wang • Yiyan Wu
}

Received: 28 May 2010 / Accepted: 25 February 2011 / Published online: 13 April 2011

(C) The Author(s) 2011. This article is published with open access at Springerlink.com

\begin{abstract}
The utilization of multiple antennas and space-time codes in multiple-input and multipleoutput (MIMO) communication systems significantly improves the transmission channel capacity without using additional bandwidth and power. The improvement is achieved by decomposing the spatial structure of transmission channels and performing appropriate temporal and spatial multiplexing. In this paper, we propose a novel theoretical framework for MIMO channel modeling and characterization in
\end{abstract}

This work was supported by the Fonds de Recherche sur la Nature et les Technologies du Québec (FQRNT) under team research project grant PR-113970.

\footnotetext{
V.-H. Pham · J.-Y. Chouinard $(\varangle) \cdot$ D. Grenier

Department of Electrical and Computer Engineering,

Université Laval, Québec, QC, Canada G1V 0A6

e-mail: jean-yves.chouinard@gel.ulaval.ca

V.-H. Pham

e-mail: phamviet@gel.ulaval.ca

D. Grenier

e-mail: dgrenier@gel.ulaval.ca

\section{H.-T. Huynh}

Bac Ha International University, Hanoi, Vietnam

e-mail: huynhhuutue@bhiu.edu.vn

\section{Wang}

Department of Electrical and Computer Engineering,

University of Western Ontario, London, ON,

Canada N6A 5B8

e-mail: wang@eng.uwo.ca

\section{$\mathrm{Y} . \mathrm{Wu}$}

Communications Research Centre Canada,

Ottawa, ON, Canada K2H 8S2

e-mail: yiyan.wu@crc.ca
}

order to facilitate the MIMO system design and performance evaluation. The channels are represented in space, time, wave vector, and frequency domains while the space-time and wave vector-frequency interdependences are considered. A realization of the theoretical framework, in a form of a practical framework, is also proposed to address the channel modeling and characterization at both transmitter and receiver sides. The utilization of the practical framework in MIMO communication system design is discussed to illustrate its applications in realistic scenarios. The angle of arrival estimation based on the proposed practical framework using field test measurement data is also presented as illustrative examples.

Keywords Spatio-temporal characterization • MIMO system design - Angle of arrival estimation • Multidimensional Fourier transform • MIMO channel characterization and modeling

\section{Introduction}

Fast growing demands of multimedia applications over mobile wireless transmission channels have raised the requirements of high transmission throughput at minimal transmission delays through fast time-varying wireless channels. The fourth generation $(4 \mathrm{G}) \mathrm{com}$ munications technologies are designed in order to response to these requirements. These communications technologies, including LTE-Advanced [1] and mobile WiMAX [2], are expected to provide the peak downlink and uplink transmission rates of 1 Gbps and 
$500 \mathrm{Mbps}$, respectively, and to maintain connections in high mobility scenarios, e.g., at $100 \mathrm{~km} / \mathrm{h}$ on highways and at $350 \mathrm{~km} / \mathrm{h}$ on bullet trains.

The multiple-input and multiple-output (MIMO) is the enabling technology for the next $4 \mathrm{G}$ broadband wireless communications systems. The key idea of the MIMO technology is to maximize the number of independent parallel sub-channels in multi-antenna communication systems by optimally decomposing the spatial structure of transmission channels, i.e., by space-time channel coding [3-7]. As a result, the channel capacity linearly increases, without consuming additional power and bandwidth, as the number of antenna elements increases $[8,9]$. This also puts channel characterization and modeling in a new context, in which rich multipath environments, e.g., Rayleigh fading channels, are preferable to communication systems.

In order to facilitate the MIMO research, the first MIMO channel models were introduced as direct extensions of single antenna, or single-input and singleoutput, channels: the sub-channels were assumed to be uncorrelated and identical-independent Rayleigh fading [10-12]. While these MIMO channel models are simple, they do not reflect the interdependent nature between the sub-channels of a MIMO channel. In an attempt to improve this drawback, the sub-channel fading correlation was modeled and its effects on channel capacity were studied [13-17].

In order to build more realistic MIMO channel models, single-directional MIMO channel models were presented. In refs. [18-20], each multipath component was represented in angle of arrival $(\phi)$, delay $(\tau)$ and observation time $(t)$, or Doppler shift $(v)$. Two channel impulse responses, including the angle-dependent time-varying channel impulse response $(h(\phi, t, \tau))$ and the multipath-Doppler spreading function $(H(\phi, v, \tau))$ were introduced. In another approach, Durgin [21] proposed to use channel impulse responses, represented in time, frequency, and space $(\tilde{h}(t, f, r))$ and in Doppler shift, delay, and wave number $(\tilde{H}(\nu, \tau, k))$ to model MIMO channels. The receiver displacement $(r)$ can be replaced by location vector $(\mathbf{r})$ and the wave number (k) can be replaced by wave vector $(\mathbf{k})$, in order to extend the results to three-dimensional (3D) space and $3 \mathrm{D}$ wave vector domains. The angle of arrival can be extracted from the received wave vector spectrum.

While the single-directional MIMO channel models characterize only the angle of arrival at the receiver, double-directional MIMO channel models include directional information at both the receiver and transmitter in order to facilitate the beamforming processing for both signal transmission and reception. In refs. [22-25], double direction implies angle of departure (AoD) and angle of arrival (AoA) while in ref. [26], it implies transmit spatial angle and receive spatial angle. The spatial angle is the antenna element separation normalized by the signal wavelength and projected on the propagation direction. Double-directional channel representation includes time-varying double-directional channel impulse response $h\left(t, \tau, \varphi_{R}, \varphi_{T}\right)$ [22,23], spatial spreading function $G\left(\theta_{R}, \theta_{T}\right)$ [26], and kernel function $h\left(\mathbf{k}_{o}, \mathbf{k}_{i}\right)$ and system function $h\left(\mathbf{k}, \mathbf{k}_{d}\right)[24,25] . \varphi_{T}$ and $\varphi_{R}$ are angle of departure and angle of arrival. $\theta_{T}$ and $\theta_{R}$ are transmit spatial angle and receive spatial angle. $\mathbf{k}_{o}$ and $\mathbf{k}_{i}$ are receive and transmit wave vector, while $\mathbf{k}$ and $\mathbf{k}_{d}$ are receive wave vector and Doppler wave vector shift. Dual MIMO channel representations in the space domain are related to double-directional MIMO channel representations through the space-wave vector duality and the 3D Fourier transform [24, 25].

The existing MIMO channel characterization and modeling techniques mentioned above have two major limitations. Firstly, channels and systems are represented in either the time domain or the space domain for simplicity and the inclusion of the other domain is assumed to be straight forward. However, there exists a relationship, and therefore an interdependence, between frequency and wave vector variables and between time and space variables. These relationships need to be taken into account in channel description and in multidimensional Fourier transform calculation. Secondly, the 3D Fourier transform is only valid for uniform plane waves. Propagating waves around the receiver can be approximated as uniform plane waves if the receiver is in the far field of the transmitter and scatterers, if any. Unfortunately, this approximation does not apply to the transmitter side. The propagating waves around the transmitter are spherical and the signal attenuation is not uniform.

In this paper, a novel MIMO channel characterization and modeling technique is proposed to overcome the two major issues of the existing characterization and modeling techniques. Firstly, a theoretical framework for MIMO channel characterization and modeling in the space, time, wave vector, and frequency domains is proposed. The space-time and wave vector-frequency interdependences are included in the framework description and the 4D Fourier transform calculation. Secondly, a practical framework, which is a realization of the theoretical framework in realistic scenarios, is proposed for MIMO channel characterization and modeling at both the transmitter and the receiver. The applications of the practical framework in designing transmit and receive MIMO antenna arrays and estimating angles of arrival are described. In addition, the presented work also finds applications in improving 
the 3D propagation channel simulation and modeling techniques, such as the one proposed in ref. [27].

The remainder of this paper is organized as follows. Section 2 recalls the fundamental concepts required for the development of the proposed framework. Section 3 discusses the wave vector-frequency spectrum estimation technique while taking into consideration the wave vector-frequency and space-time relationships. Section 4 introduces the space-time and wave vectorfrequency compound impulses by applying the multidimensional Dirac impulse concept in the 4D spacetime and 4D wave vector-frequency domains. Section 5 recalls the kernel function definitions. Sections 6 and 7 present the proposed theoretical and practical frameworks. Sections 8 and 9 discuss the framework applications in MIMO antenna array design and AoA estimation. Section 10 concludes the paper.

\section{Background}

\subsection{Wave vector}

Wave vector is a vector representation of radio waves. The direction of a wave vector indicates the wave propagation direction and the magnitude is the angular wave number. The magnitude of the wave vector $\mathbf{k}$ is:

$$
|\mathbf{k}|=\frac{2 \pi}{\lambda}=\frac{\omega}{v_{p}}
$$

where $\lambda$ is the wavelength, $\omega$ is the angular frequency, and $v_{p}$ is the propagation velocity.

Let $k_{x}, k_{y}$, and $k_{z}$ be the three components of vector $\mathbf{k}$, then:

$$
\begin{gathered}
k_{x}=\frac{2 \pi}{\lambda_{x}}=\frac{\omega}{v_{p x}} \\
k_{y}=\frac{2 \pi}{\lambda_{y}}=\frac{\omega}{v_{p y}} \\
k_{z}=\frac{2 \pi}{\lambda_{z}}=\frac{\omega}{v_{p z}},
\end{gathered}
$$

where $\lambda_{x}, \lambda_{y}$, and $\lambda_{z}$ are the wavelengths and $v_{p x}, v_{p y}$, and $v_{p z}$ are the phase velocities along the $x$-, $y$-, and $z$-axes, respectively. ${ }^{1}$

\footnotetext{
${ }^{1}$ It is important not to confuse phase velocities $v_{p x}, v_{p y}$, and $v_{p z}$ with the projections of the propagation velocity vector $\mathbf{v}_{\mathbf{p}}$ onto axes $x, y$, and $z$.
}

\subsection{Plane waves}

A wave is said to be planar if its wave fronts are plane in the space domain. If the wave amplitudes on a wave plane are constants, then the wave is a uniform plane wave. An example of a uniform plane wave is the electromagnetic wave radiated from an infinite current sheet. Plane waves do not exist in practice, but they provide a simple framework for the study of electromagnetic wave radiation and propagation. Furthermore, plane waves can be used as an approximation of propagation waves in real physical scenarios.

\subsection{D Fourier transform}

A location in the three-dimensional (3D) space is represented by a vector, called space vector, or location/position vector (r). In the Cartesian coordinates system, space vector $\mathbf{r}$ has three components, denoted as $r_{x}, r_{y}$, and $r_{z}$. A duality exists between time and frequency [28]. For a uniform plane wave, the duality exists also between space vector and wave vector [24] through the 3D Fourier transform. The 3D Fourier transform converts a signal in the spatial domain into the wave vector domain. Alternatively, the $3 \mathrm{D}$ inverse Fourier transform converts a signal in the wave vector domain into the spatial domain. The continuous 3D Fourier and inverse Fourier transforms are:

$$
\begin{aligned}
& S(\mathbf{k})=\int s(\mathbf{r}) e^{j \mathbf{r} \cdot \mathbf{k}} \mathrm{d} \mathbf{r} \\
& s(\mathbf{r})=\frac{1}{(2 \pi)^{3}} \int S(\mathbf{k}) e^{-j \mathbf{k} \cdot \mathbf{r}} \mathrm{d} \mathbf{k},
\end{aligned}
$$

where $\mathrm{d} \mathbf{k}$ represents $\mathrm{d} k_{x} \mathrm{~d} k_{y} \mathrm{~d} k_{z}$ and $\mathrm{d} \mathbf{r}$ represents $\mathrm{d} r_{x} \mathrm{~d} r_{y} \mathrm{~d} r_{z}$.

\subsection{Wave vector-frequency and space-time relationships}

Wave vector and frequency have a direct relationship: $|\mathbf{k}|=\frac{2 \pi}{\lambda}=\frac{\omega}{v_{p}}=\frac{2 \pi f}{v_{p}}$. In Eq. 4, the signal wave vector spectrum component at wave vector $\mathbf{k}$ transmitted from the space origin is $S(\mathbf{k})$. As the signal reaches location $\mathbf{r}$, the signal component at wave vector $\mathbf{k}$ is $S(\mathbf{k}) e^{-j \mathbf{k} \cdot \mathbf{r}}$. One observes that no attenuation affects the signal and that no changes occurred to wave vector $\mathbf{k}$ in the calculation of the three-dimensional Fourier transform as a lossless and scatter-free environment is assumed. In this environment, ${ }^{2}$ the time variable $(t)$ is the ratio

\footnotetext{
${ }^{2}$ The signal is assumed to be transmitted from the spatial origin, $\mathbf{0}=(0,0,0)$. Refer to Section 4.3 for detailed discussion on the space-time relationship.
} 
between the magnitude of location vector $|\mathbf{r}|$ and the propagation velocity $\left(v_{p}\right): t=\frac{|\mathbf{r}|}{v_{p}}$.

\section{Wave vector-frequency spectrum estimation}

\subsection{Four-dimensional compound Fourier transform}

The wave vector-frequency spectrum is the compound transform of a 3D Fourier transform with respect to the space variable, $\mathcal{F}_{\mathbf{r}}(\cdot)$, and a Fourier transform with respect to the time variable, $\mathcal{F}_{t}(\cdot)$ :

$$
\begin{aligned}
S(f ; \mathbf{k}) & =\iint s(t ; \mathbf{r}) e^{-j 2 \pi f t} e^{j \mathbf{k} \cdot \mathbf{r}} \mathrm{d} t \mathrm{~d} \mathbf{r} \\
s(t ; \mathbf{r}) & =\frac{1}{(2 \pi)^{3}} \iint S(f ; \mathbf{k}) e^{j 2 \pi f t} e^{-j \mathbf{k} \cdot \mathbf{r}} \mathrm{d} f \mathrm{~d} \mathbf{k} .
\end{aligned}
$$

Frequency and wave vectors are related to each other through the wave number. Also, the location vector and the time are related to each other through the propagation velocity, as discussed in Section 2.4. This means that the integrals in Eqs. 5 and 6 are not performed all over the 4D hyperspace, i.e., 3D space and time in Eq. 5 and 3D wave vector and frequency in Eq. 6, but rather on a 4D hypersurface. Equations 5 and 6 can be rewritten as:

$$
\begin{aligned}
S(f ; \mathbf{k}) & =\int_{H_{s_{-}}} s(t ; \mathbf{r}) e^{-j 2 \pi f t} e^{j \mathbf{k} \cdot \mathbf{r}} \mathrm{d} h_{s_{-} t} \\
s(t ; \mathbf{r}) & =\frac{1}{(2 \pi)^{3}} \int_{H_{w_{-}}} S(f ; \mathbf{k}) e^{j 2 \pi f t} e^{-j \mathbf{k} \cdot \mathbf{r}} \mathrm{d} h_{w_{-} f},
\end{aligned}
$$

where $H_{s_{-} t}$ is the integration hypersurface, or integration domain, defined by the space-time relationship, $t=\frac{|\mathbf{r}|}{v_{p}}$, and $H_{w_{-} f}$ is the integration hypersurface defined by the wave vector-frequency relationship, $|\mathbf{k}|=\frac{2 \pi f}{v_{p}}$. Hypersurfaces $H_{w_{-} f}$ and $H_{s_{-} t}$ are dependent on the transmission media characteristics, i.e., propagation velocity $v_{p}$. From Eqs. 7 and 8, we have Eqs. 9 and 10 .

Equations 9 and 10 are convenient in the mathematical sense as they describe explicitly the space-time and wave vector-frequency relationships in a general form. If one needs to modify the space-time and/or wave vector-frequency relationships, Eqs. 9 and 10 still remain unchanged.

Another approach to the compound Fourier transform calculation is to represent the time variable from the space vector, i.e., $t=\frac{|\mathbf{r}|}{v_{p}}$, and the frequency variable from the wave vector, i.e., $f=\frac{|\mathbf{k}| v_{p}}{2 \pi}$. Assuming that $\frac{1}{v_{p}^{2}} \ll 1$ and $\frac{v_{p}^{2}}{4 \pi^{2}} \gg 1$, Eqs. 7 and 8 become Eqs. 11 and 12 .

$$
\begin{aligned}
S(f ; \mathbf{k})= & \int s(t ; \mathbf{r}(t)) e^{-j 2 \pi f t} e^{j \mathbf{k} \cdot \mathbf{r}(t)} \\
& \times\left[1+\left(\frac{\mathrm{d} r_{x}}{\mathrm{~d} t}\right)^{2}+\left(\frac{\mathrm{d} r_{y}}{\mathrm{~d} t}\right)^{2}+\left(\frac{\mathrm{d} r_{z}}{\mathrm{~d} t}\right)^{2}\right]^{1 / 2} d t
\end{aligned}
$$

$$
\begin{aligned}
s(t ; \mathbf{r})= & \frac{1}{(2 \pi)^{3}} \int S(f ; \mathbf{k}) e^{j 2 \pi f t} e^{-j \mathbf{k} \cdot \mathbf{r}} \\
& \times\left[1+\left(\frac{\mathrm{d} k_{x}}{\mathrm{~d} f}\right)^{2}+\left(\frac{\mathrm{d} k_{y}}{\mathrm{~d} f}\right)^{2}+\left(\frac{\mathrm{d} k_{z}}{\mathrm{~d} f}\right)^{2}\right]^{1 / 2} \mathrm{~d} f
\end{aligned}
$$

$$
\begin{aligned}
\left.S(f ; \mathbf{k})\right|_{f=\frac{\mid \mathbf{k} v v_{p}}{2 \pi}} & \int s\left(t=\frac{|\mathbf{r}|}{v_{p}} ; \mathbf{r}\right) e^{-j 2 \pi f \frac{|\mathbf{r}|}{v_{p}}} e^{j \mathbf{k} \cdot \mathbf{r}} \\
& \times\left[1+\left(\frac{\partial t}{\partial r_{x}}\right)^{2}+\left(\frac{\partial t}{\partial r_{y}}\right)^{2}+\left(\frac{\partial t}{\partial r_{z}}\right)^{2}\right]^{1 / 2} \mathrm{~d} \mathbf{r} \\
= & \int s\left(t=\frac{|\mathbf{r}|}{v_{p}} ; \mathbf{r}\right) e^{-j 2 \pi f \frac{|r|}{v_{p}}} e^{j \mathbf{k} \cdot \mathbf{r}} \\
& \times\left[1+\frac{1}{v_{p}^{2}} \frac{r_{x}^{2}+r_{y}^{2}+r_{z}^{2}}{r_{x}^{2}+r_{y}^{2}+r_{z}^{2}}\right]^{1 / 2} \mathrm{~d} \mathbf{r} \\
= & \int s\left(t=\frac{|\mathbf{r}|}{v_{p}} ; \mathbf{r}\right) e^{-j 2 \pi f \frac{|r|}{v_{p}}} e^{j \mathbf{k} \cdot \mathbf{r}} \\
& \times\left[1+\frac{1}{v_{p}^{2}}\right]^{1 / 2} \mathrm{~d} \mathbf{r} \\
\approx & \int s\left(t=\frac{|\mathbf{r}|}{v_{p}} ; \mathbf{r}\right) e^{-j 2 \pi f \frac{|\mathbf{r}|}{v_{p}}} e^{j \mathbf{k} \cdot \mathbf{r}} \mathrm{d} \mathbf{r}
\end{aligned}
$$

$$
\begin{aligned}
\left.s(t ; \mathbf{r})\right|_{t=\frac{|\mathbf{r}|}{v_{p}}=} & \frac{1}{(2 \pi)^{3}} \int S\left(f=\frac{|\mathbf{k}| v_{p}}{2 \pi} ; \mathbf{k}\right) e^{j|\mathbf{k}| v_{p} t} e^{-j \mathbf{k} \cdot \mathbf{r}} \\
& \times\left[1+\left(\frac{\partial f}{\partial k_{x}}\right)^{2}+\left(\frac{\partial f}{\partial k_{y}}\right)^{2}+\left(\frac{\partial f}{\partial k_{z}}\right)^{2}\right]^{1 / 2} \mathrm{~d} \mathbf{k} \\
= & \frac{1}{(2 \pi)^{3}} \int S\left(f=\frac{|\mathbf{k}| v_{p}}{2 \pi} ; \mathbf{k}\right) e^{j \mathbf{k} \mid v_{p} t} e^{-j \mathbf{k} \cdot \mathbf{r}} \\
& \times\left[1+\frac{v_{p}^{2}}{4 \pi^{2}} \frac{k_{x}^{2}+k_{y}^{2}+k_{z}^{2}}{k_{x}^{2}+k_{y}^{2}+k_{z}^{2}}\right]^{1 / 2} \mathrm{~d} \mathbf{k}
\end{aligned}
$$




$$
\begin{aligned}
= & \frac{1}{(2 \pi)^{3}} \int S\left(f=\frac{|\mathbf{k}| v_{p}}{2 \pi} ; \mathbf{k}\right) e^{j|\mathbf{k}| v_{p} t} e^{-j \mathbf{k} \cdot \mathbf{r}} \\
& \times\left[1+\frac{v_{p}^{2}}{4 \pi^{2}}\right]^{1 / 2} \mathrm{~d} \mathbf{k} \\
\approx & \frac{v_{p}}{(2 \pi)^{4}} \int S\left(f=\frac{|\mathbf{k}| v_{p}}{2 \pi} ; \mathbf{k}\right) e^{j|\mathbf{k}| v_{p} t} e^{-j \mathbf{k} \cdot \mathbf{r}} \mathrm{d} \mathbf{k}
\end{aligned}
$$

\section{Space, wave vector, and compound impulses}

\subsection{Impulse function definitions}

In order to characterize the channel and system in any $\mathrm{N}$-dimensional space, we need to use multidimensional Dirac impulses [29, pp. 23-24], [30, pp. 26-29]. Let $\mathbf{x}$ be an $N$-dimensional vector defined in $\mathbb{R}^{N}$. We generalize an $N$-dimensional Dirac impulse as follows:

$$
\underbrace{\int \cdots \int}_{N} f(\mathbf{x}) \delta\left(\mathbf{x}-\mathbf{x}_{0}\right) \mathrm{d} x_{1} \mathrm{~d} x_{2} \cdots \mathrm{d} x_{N}=f\left(\mathbf{x}_{\mathbf{0}}\right),
$$

where $f(\mathbf{x})$ is a function defined over $\mathbb{R}^{N}$, and $x_{1}, x_{2}, \ldots, x_{N}$ are the components of vector $\mathbf{x}$.

In the following, $\mathbf{x}$ might be a combination of vectors and variables $\mathbf{r}, \mathbf{k}, f$ and $t . \delta(\mathbf{r}), \delta(\mathbf{k}), \delta(\mathbf{r}, t)$, $\delta(\mathbf{r}, f), \delta(\mathbf{k}, t)$, and $\delta(\mathbf{k}, f)$ are the multidimensional Dirac impulses in space, wave vector, space-time, space-frequency, wave vector-time, and wave vectorfrequency, respectively.

\subsection{Space vector-wave vector duality}

Applying the 3D Fourier transform on the space impulse function, $\delta(\mathbf{r})$, gives $\mathcal{F}_{\mathbf{r}}\{\delta(\mathbf{r})\}=1$. Therefore, the wave vector spectrum of the space impulse is equal to unity and is independent of the wave vector. Similarly, the inverse 3D Fourier transform of the wave vector impulse function at wave vector $\mathbf{k}_{0}$ is a complex exponential with equal unity magnitude: $\mathcal{F}_{\mathbf{k}}^{-1}\left\{\delta\left(\mathbf{k}-\mathbf{k}_{0}\right)\right\}=e^{-j \mathbf{k}_{0} \cdot \mathbf{r}}$. This means that an impulse at frequency $f_{0}$ emitted in the direction of $\mathbf{k}_{0}$ will propagate all over the space with equal (unit) magnitude. This is due to the assumption of uniform plane wave propagation.

A frequency impulse, $\delta\left(f-f_{0}\right)$, is white in time domain and a time impulse, $\delta(t)$, is white in frequency domain. This duality can be transposed to space and wave vector domains through the 3D Fourier transform. The wave vector impulse is white in the tri-dimensional space domain and the spatial impulse is white in the 3D wave vector domain. Due to the existing relationships between wave vector and frequency, and between location vector and time (Eqs. 11 and 6), the wave vector impulse, $\delta(\mathbf{k})$, is white in space and also white in time; the space impulse, $\delta(\mathbf{r})$, is white in wave vector and also in frequency. Therefore, a four-dimensional wave vector-time impulse, $\delta(\mathbf{k}, t)$, and a $4 \mathrm{D}$ space-frequency impulse, $\delta(\mathbf{r}, f)$, do not have physical sense.

\subsection{Space-time relationship}

Here, we review the space-time relationship using the multidimensional impulse functions. The space impulse at location $\mathbf{r}_{0}, \delta\left(\mathbf{r}-\mathbf{r}_{0}\right)$, is white in wave vector and in frequency. A signal that is white in frequency implies that it is a time impulse. We need to determine the time instant that the impulse occurred in order to find the space-time relationship. The wave vector spectrum of the space impulse at location $\mathbf{r}_{0}, \delta\left(\mathbf{r}-\mathbf{r}_{0}\right)$, is:

$\mathcal{F}_{\mathbf{r}}\left\{\delta\left(\mathbf{r}-\mathbf{r}_{0}\right)\right\}=e^{j \mathbf{r}_{0} \cdot \mathbf{k}}=e^{j\left|\mathbf{r}_{0}\right||\mathbf{k}| \cos \angle \mathbf{r}_{0}, \mathbf{k}}$.

Representing the wave number, $|\mathbf{k}|$, through frequency, the wave vector spectrum becomes:

$$
\begin{aligned}
\mathcal{F}_{\mathbf{r}}\left\{\delta\left(\mathbf{r}-\mathbf{r}_{0}\right)\right\} & =e^{j \frac{2 \pi f}{v_{p}}\left|\mathbf{r}_{0}\right| \cos \angle \mathbf{r}_{0}, \mathbf{k}} \\
& =e^{j 2 \pi f \cos \angle \mathbf{r}_{0}, \mathbf{k} \frac{\left|\mathbf{r}_{0}\right|}{v_{p}}} .
\end{aligned}
$$

Examining Eq. 15 along a given propagation direction, i.e., angle $\angle \mathbf{r}_{0}, \mathbf{k}$ is set, the spectrum in the frequency domain of a spatial impulse is $e^{j 2 \pi f^{\prime} t_{0}}$, with $f^{\prime}=$ $f \cos \angle \mathbf{r}_{0}, \mathbf{k}$ and $t_{0}=\frac{\left|\mathbf{r}_{0}\right|}{v_{p}}$. This is the spectrum of a time impulse at time instant $t=t_{0}$. This shows clearly the space-time relationship referred to in Section 2.4. Similarly, assuming space-time relationship $t=\frac{|\mathbf{r}|}{v_{p}}$, then the wave vector-frequency relationship $|\mathbf{k}|=\frac{2 \pi f}{v_{p}}$ is derived. This is another form of duality in space-time and wave vector-frequency domains: the wave vectorfrequency relationship assumes the space-time relationship and vice versa.

\subsection{Space-time impulse functions}

The space-time impulse function, $\delta(\mathbf{r}, t)$, exists at the spatial origin, $\mathbf{r}=\mathbf{0}$, and at the time origin, $t=0$. Due to the space-time relationship mentioned above, a space impulse is also a space-time compound impulse. Apply- 
ing Eq. 11, the 4D spectrum of a space-time compound impulse at position $\mathbf{r}_{0}$ and time $t_{0}$ is:

$$
\begin{aligned}
& \left.\mathcal{F}_{\mathbf{r}, t}\left\{\delta\left(\mathbf{r}-\mathbf{r}_{0}, t-t_{0}\right)\right\}\right|_{t=\frac{\mid \mathbf{r}}{v_{p}}, t_{0}=\frac{\left|\mathbf{r}_{0}\right|}{v_{p}}} \\
& =\left.\int \delta\left(\mathbf{r}-\mathbf{r}_{0}, t-t_{0}\right) e^{j \mathbf{k} \cdot \mathbf{r}} e^{-j 2 \pi f t} \mathrm{~d} \mathbf{r}\right|_{|\mathbf{k}|=\frac{2 \pi f}{v_{p}}} \\
& =\left.e^{j \mathbf{k} \cdot \mathbf{r}_{0}} e^{-j 2 \pi f t_{0}}\right|_{|\mathbf{k}|=\frac{2 \pi f}{v_{p}}}
\end{aligned}
$$

\subsection{Wave vector-frequency impulse functions}

The wave vector impulse function $(\delta(\mathbf{k}))$ occurs only when the wave vector equals to wave vector zero $(\mathbf{k}=$ 0). In practice, we usually refer to an impulse at wave vector $\mathbf{k}_{0}$ rather than at wave vector zero, as for a frequency impulse at frequency $f_{0}, \delta\left(f-f_{0}\right)$. Due to the wave vector-frequency relationship, a wave vector impulse $\delta\left(\mathbf{k}-\mathbf{k}_{0}\right)$ is also a wave vector-frequency compound impulse at wave vector $\mathbf{k}_{0}$ and frequency $f_{0}$. In space and time, a wave vector-frequency impulse is a complex exponential at frequency $f_{0}$ propagating in the direction of $\mathbf{k}_{0}$. Applying Eq. 12, the space-time representation of a wave vector-frequency compound impulse is found:

$$
\begin{aligned}
& \left.\mathcal{F}_{\mathbf{k}, f}^{-1}\left\{\delta\left(\mathbf{k}-\mathbf{k}_{0}, f-f_{0}\right)\right\}\right|_{|\mathbf{k}|=\frac{2 \pi f}{v_{p}},\left|\mathbf{k}_{0}\right|=\frac{2 \pi f_{0}}{v_{p}}} \\
& =\left.\frac{v_{p}}{(2 \pi)^{4}} \int \delta\left(\mathbf{k}-\mathbf{k}_{0}, f-f_{0}\right) e^{-j \mathbf{k} \cdot \mathbf{r}} e^{j 2 \pi f t} \mathrm{~d} \mathbf{k}\right|_{t=\frac{|\mathbf{r}|}{v_{p}}} \\
& =\left.\frac{v_{p}}{(2 \pi)^{4}} e^{-j \mathbf{k}_{0} \cdot \mathbf{r}} e^{j 2 \pi f_{0} t}\right|_{t=\frac{|\mathbf{r}|}{v_{p}}}
\end{aligned}
$$

\section{Spatial kernel functions}

\subsection{Definition of the spatial kernel functions}

Here, for simplicity, we consider only the space and wave vector variables in signal and system representation. Time and frequency variables will be included, when it is necessary, in order to derive the complete signal and system characterization. In a communications system, the transmitted and received signals can be represented in either space or wave vector domains. A communications system transforms the transmitted signals represented in either space or wave vector, into received signals, either in space or wave vector domains. Four operators are defined to form the kernel functions: $K_{s s}\left(\mathbf{r}_{o}, \mathbf{r}_{i}\right), K_{w w}\left(\mathbf{k}_{o}, \mathbf{k}_{i}\right), K_{s w}\left(\mathbf{r}_{o}, \mathbf{k}_{i}\right)$, and $K_{w s}\left(\mathbf{k}_{o}, \mathbf{r}_{i}\right) . \mathbf{r}_{i}$ and $\mathbf{r}_{o}$ are the positions of the transmitter and the receiver, respectively, while $\mathbf{k}_{i}$ and $\mathbf{k}_{o}$ are the transmit and the receive wave vectors, respectively. These kernels functions are derived directly from Bello's results [31] in time and frequency domains, and developed by Guguen and Zein [24, 25] for spatial channel impulse responses. The relationships between the transmitted and received signals using these four kernel functions are:

$$
\begin{aligned}
s_{o}\left(\mathbf{r}_{o}\right) & =\int s_{i}\left(\mathbf{r}_{i}\right) K_{s s}\left(\mathbf{r}_{o}, \mathbf{r}_{i}\right) \mathrm{d} \mathbf{r}_{i} \\
S_{o}\left(\mathbf{k}_{o}\right) & =\int S_{i}\left(\mathbf{k}_{i}\right) K_{w w}\left(\mathbf{k}_{o}, \mathbf{k}_{i}\right) \mathrm{d} \mathbf{k}_{i} \\
s_{o}\left(\mathbf{r}_{o}\right) & =\int S_{i}\left(\mathbf{k}_{i}\right) K_{s w}\left(\mathbf{r}_{o}, \mathbf{k}_{i}\right) \mathrm{d} \mathbf{k}_{i} \\
S_{o}\left(\mathbf{k}_{o}\right) & =\int s_{i}\left(\mathbf{r}_{i}\right) K_{w s}\left(\mathbf{k}_{o}, \mathbf{r}_{i}\right) \mathrm{d} \mathbf{r}_{i},
\end{aligned}
$$

where $d \mathbf{r}_{i}=d r_{i x} d r_{i y} d r_{i z}$ and $d \mathbf{k}_{i}=d k_{i x} d k_{i y} d k_{i z} \cdot s\left(\mathbf{r}_{i}\right)$ and $S\left(\mathbf{k}_{i}\right)$ are the transmitted signal in 3D spatial domain and transmitted signal spectrum in wave vector domain respectively, $s\left(\mathbf{r}_{o}\right)$ and $S\left(\mathbf{k}_{o}\right)$ are the received signal in the 3D spatial domain and the received signal spectrum in wave vector domain.

\subsection{Spatial kernel function relationships}

Applying the Fourier transform on Eq. 18, the equality becomes:

$S_{o}\left(\mathbf{k}_{o}\right)=\int s_{i}\left(\mathbf{r}_{i}\right) \mathcal{F}_{\mathbf{r}_{o}}\left\{K_{s S}\left(\mathbf{r}_{o}, \mathbf{r}_{i}\right)\right\} \mathrm{d} \mathbf{r}_{i}$

where $\mathcal{F}_{\mathbf{r}_{o}}\left\{K_{S S}\left(\mathbf{r}_{o}, \mathbf{r}_{i}\right)\right\}$ is the Fourier transform of function $K_{s s}\left(\mathbf{r}_{o}, \mathbf{r}_{i}\right)$, with respect to space variable $\mathbf{r}_{o}$. Comparing this equation and Eq. 21, one obtains the following relationships:

$$
\begin{aligned}
K_{w s}\left(\mathbf{k}_{o}, \mathbf{r}_{i}\right) & =\mathcal{F}_{\mathbf{r}_{o}}\left\{K_{s S}\left(\mathbf{r}_{o}, \mathbf{r}_{i}\right)\right\} \\
K_{s s}\left(\mathbf{r}_{o}, \mathbf{r}_{i}\right) & =\mathcal{F}_{\mathbf{k}_{o}}^{-1}\left\{K_{w s}\left(\mathbf{k}_{o}, \mathbf{r}_{i}\right)\right\} .
\end{aligned}
$$

Similarly, applying the Fourier transform on Eq. 20 and comparing with Eq. 19, the relationship between kernel function $K_{w w}(\cdot)$ and kernel function $K_{s w}(\cdot)$ is obtained:

$$
\begin{aligned}
K_{w w}\left(\mathbf{k}_{o}, \mathbf{k}_{i}\right) & =\mathcal{F}_{\mathbf{r}_{o}}\left\{K_{s w}\left(\mathbf{r}_{o}, \mathbf{k}_{i}\right)\right\} \\
K_{s w}\left(\mathbf{r}_{o}, \mathbf{k}_{i}\right) & =\mathcal{F}_{\mathbf{k}_{o}}^{-1}\left\{K_{w w}\left(\mathbf{k}_{o}, \mathbf{k}_{i}\right)\right\} .
\end{aligned}
$$

In order to determine the relationship between kernel functions $K_{w s}\left(\mathbf{k}_{o}, \mathbf{r}_{i}\right)$ and $K_{w w}\left(\mathbf{k}_{o}, \mathbf{k}_{i}\right)$, the input sig- 


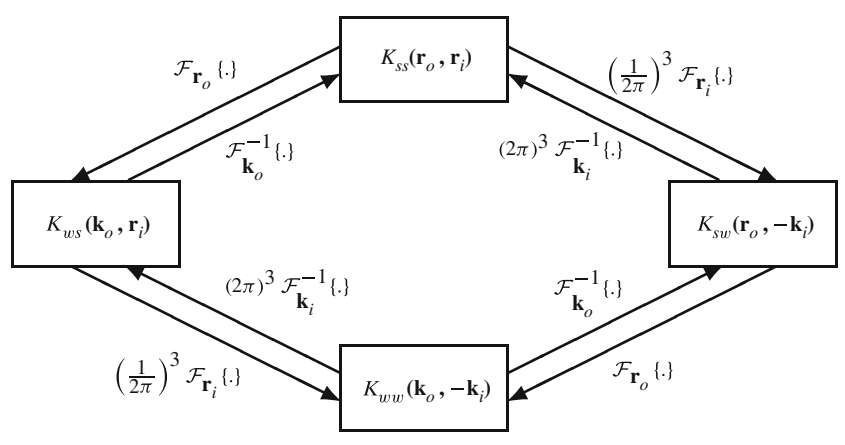

Fig. 1 Relationships between the kernel functions

nal $S_{i}\left(\mathbf{k}_{i}\right)$ in Eq. 19 is replaced by the Fourier transform of its dual function, i.e., $s_{i}\left(\mathbf{r}_{i}\right)$ :

$$
\begin{aligned}
S_{o}\left(\mathbf{k}_{o}\right) & =\int\left\{\int s_{i}\left(\mathbf{r}_{i}\right) e^{j \mathbf{r}_{i} \cdot \mathbf{k}_{i}} \mathrm{~d} \mathbf{r}_{i}\right\} K_{w w}\left(\mathbf{k}_{o}, \mathbf{k}_{i}\right) \mathrm{d} \mathbf{k}_{i} \\
& =\int s_{i}\left(\mathbf{r}_{i}\right)\left\{\int K_{w w}\left(\mathbf{k}_{o}, \mathbf{k}_{i}\right) e^{j \mathbf{r}_{i} \cdot \mathbf{k}_{i}} \mathrm{~d} \mathbf{k}_{i}\right\} \mathrm{d} \mathbf{r}_{i} .
\end{aligned}
$$

Comparing this equation and Eq. 21, leads to:

$$
\begin{aligned}
K_{w s}\left(\mathbf{k}_{o}, \mathbf{r}_{i}\right) & =\int K_{w w}\left(\mathbf{k}_{o}, \mathbf{k}_{i}\right) e^{j \mathbf{r}_{i} \cdot \mathbf{k}_{i}} \mathrm{~d} \mathbf{k}_{i} \\
& =(2 \pi)^{3} \mathcal{F}_{\mathbf{k}_{i}}^{-1}\left\{K_{w w}\left(\mathbf{k}_{o},-\mathbf{k}_{i}\right)\right\}
\end{aligned}
$$

or

$K_{w w}\left(\mathbf{k}_{o},-\mathbf{k}_{i}\right)=\left(\frac{1}{2 \pi}\right)^{3} \mathcal{F}_{\mathbf{r}_{i}}\left\{K_{w s}\left(\mathbf{k}_{o}, \mathbf{r}_{i}\right)\right\}$.

Applying similar reasoning to Eqs. 20 and 18, the relationships between kernel functions $K_{s s}\left(\mathbf{r}_{o}, \mathbf{r}_{i}\right)$ and $K_{s w}\left(\mathbf{r}_{o}, \mathbf{k}_{i}\right)$ are obtained:

$$
\begin{aligned}
K_{s w}\left(\mathbf{r}_{o},-\mathbf{k}_{i}\right) & =\left(\frac{1}{2 \pi}\right)^{3} \mathcal{F}_{\mathbf{r}_{i}}\left\{K_{s S}\left(\mathbf{r}_{o}, \mathbf{r}_{i}\right)\right\} \\
K_{s s}\left(\mathbf{r}_{o}, \mathbf{r}_{i}\right) & =(2 \pi)^{3} \mathcal{F}_{\mathbf{k}_{i}}^{-1}\left\{K_{s w}\left(\mathbf{r}_{o},-\mathbf{k}_{i}\right)\right\} .
\end{aligned}
$$

The above relationships are summarized in Fig. 1.

\section{Spatio-temporal channel characterization-theoretical framework}

\subsection{Space-time kernel functions}

In Section 5, we omitted the time and frequency variables from the signal and system representation. A complete system representation includes the variables in time, frequency, space and wave vector domains. Due to the time-frequency duality and the space-wave vector duality, the input and output signals of a communications system are represented as the functions of two variables, i.e., time or frequency variable, and space or wave vector variable. There are four combinations of these two variables: space-time, space-frequency, wave vector-time and wave vector-frequency. Following the same approach in Section 5, we defined a set of 16 kernel functions, as listed in Table 1. This set of kernel functions is complete in the sense that it characterizes systems and channels in all the related domains and the space-time and wave vector-frequency relationships are included implicitly. The relationships between the space-time kernel functions and their applications in practical contexts are discussed in subsequent paragraphs.

\subsection{Space-time kernel function relationships}

Following the same reasoning as in Section 5, kernel functions in space-time-wave vector-frequency are also interconnected to each other by the multidimensional Fourier transform. These relationships are summarized in Fig. 2. Note that, in this figure, the Fourier transform on the input spatial vector $\left(\mathbf{r}_{i}\right)$ is multiplied by $\left(\frac{1}{2 \pi}\right)^{3}$ while the inverse Fourier transform on the input wave vector $\left(\mathbf{k}_{i}\right)$ is multiplied by $(2 \pi)^{3}$. In Fig. 2, we also need to assign a negative sign to either the input time $\left(t_{i}\right)$ and the input location vector $\left(\mathbf{r}_{i}\right)$, or the input frequency $\left(f_{i}\right)$ and the input wave vector $\left(\mathbf{k}_{i}\right)$. The 16 kernel functions are represented by the 16 vertices of two cubes: the vertex label numbers are the labels of the corresponding kernel functions defined in Table 1. The bold lines represent the 3D spacewave vector Fourier transform and inverse 3D Fourier transform pair while the thin lines represent the timefrequency Fourier transform and inverse Fourier transform pair. One observes that the 16 kernel functions are grouped into four groups of four functions: these are $\left(K_{1}, K_{2}, K_{3}, K_{4}\right),\left(K_{5}, K_{6}, K_{7}, K_{8}\right),\left(K_{9}, K_{10}, K_{11}, K_{12}\right)$, and $\left(K_{13}, K_{14}, K_{15}, K_{16}\right)$. The functions from the same

Table 1 Ensemble of Kernel functions in space-time-wave vector-frequency

\begin{tabular}{llcl}
\hline No. & Kernel function & No. & Kernel function \\
\hline 1 & $K_{1}\left(\mathbf{r}_{\mathbf{o}}, \mathbf{r}_{\mathbf{i}} ; t_{o}, t_{i}\right)$ & 9 & $K_{9}\left(\mathbf{r}_{\mathbf{o}}, \mathbf{r}_{\mathbf{i}} ; f_{o}, f_{i}\right)$ \\
2 & $K_{2}\left(\mathbf{r}_{\mathbf{o}}, \mathbf{k}_{\mathbf{i}} ; t_{o}, t_{i}\right)$ & 10 & $K_{10}\left(\mathbf{r}_{\mathbf{o}}, \mathbf{k}_{\mathbf{i}} ; f_{o}, f_{i}\right)$ \\
3 & $K_{3}\left(\mathbf{k}_{\mathbf{o}}, \mathbf{k}_{\mathbf{i}} ; t_{o}, t_{i}\right)$ & 11 & $K_{11}\left(\mathbf{k}_{\mathbf{o}}, \mathbf{k}_{\mathbf{i}} ; f_{o}, f_{i}\right)$ \\
4 & $K_{4}\left(\mathbf{k}_{\mathbf{o}}, \mathbf{r}_{\mathbf{i}} ; t_{o}, t_{i}\right)$ & 12 & $K_{12}\left(\mathbf{k}_{\mathbf{o}}, \mathbf{r}_{\mathbf{i}} ; f_{o}, f_{i}\right)$ \\
5 & $K_{5}\left(\mathbf{r}_{\mathbf{o}}, \mathbf{r}_{\mathbf{i}} ; t_{o}, f_{i}\right)$ & 13 & $K_{13}\left(\mathbf{r}_{\mathbf{o}}, \mathbf{r}_{\mathbf{i}} ; f_{o}, t_{i}\right)$ \\
6 & $K_{6}\left(\mathbf{r}_{\mathbf{o}}, \mathbf{k}_{\mathbf{i}} ; t_{o}, f_{i}\right)$ & 14 & $K_{14}\left(\mathbf{r}_{\mathbf{o}}, \mathbf{k}_{\mathbf{i}} ; f_{o}, t_{i}\right)$ \\
7 & $K_{7}\left(\mathbf{k}_{\mathbf{o}}, \mathbf{k}_{\mathbf{i}} ; t_{o}, f_{i}\right)$ & 15 & $K_{15}\left(\mathbf{k}_{\mathbf{o}}, \mathbf{k}_{\mathbf{i}} ; f_{o}, t_{i}\right)$ \\
8 & $K_{8}\left(\mathbf{k}_{\mathbf{o}}, \mathbf{r}_{\mathbf{i}} ; t_{o}, f_{i}\right)$ & 16 & $K_{16}\left(\mathbf{k}_{\mathbf{o}}, \mathbf{r}_{\mathbf{i}} ; f_{o}, t_{i}\right)$ \\
\hline
\end{tabular}




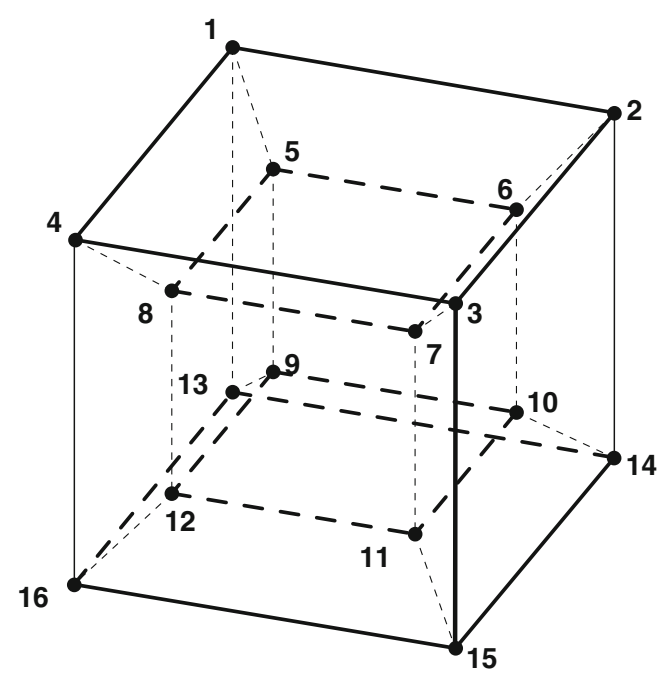

Fig. 2 Complete kernel function relationship

group have a common time-frequency variable pair and lie on the same surface, which is parallel to the surface formed by four points $(1,2,3,4)$. They are interconnected by the $3 \mathrm{D}$ Fourier transform. These four groups are interconnected by the time-frequency Fourier transform pairs as shown by the thin lines at the edge of the cubes. Solid and dashed lines are used to show the external and the internal views of the kernel function relationship cube.

\section{Spatio-temporal channel characterization-practical framework}

The transposition of the spatio-temporal theoretical framework to practical wireless communication scenarios is not straightforward since propagating waves have some degree of curvature. In the far field [32] of the transmitter, the curvature becomes less significant and, within a bounded region, the propagating waves can be regarded approximatively as plane waves. In ref. [25], Guguen and Zein presented the utilization of the spatial kernel functions in characterizing MIMO channel for indoor WLAN applications. Unfortunately, the plane wave approximation is not always valid in indoor scenarios, since the receivers are not always in the far field of scatterers, e.g., walls and furniture.

In this work, the plane wave approximation is made to find applications of the proposed framework to realistic scenarios at receiver side. However, this approximation cannot be used at transmitter side since the space region around the transmitter does not satisfy the far field criterion. The approach used for applying the framework at transmitter side will be discussed in Section 7.2.

\subsection{Application of the modeling framework at the receiver side}

When one considers uniform plane wave propagation, it is usually assumed that the receiver is located very far from the transmitter. In order to apply the proposed framework at the receiver in practical contexts, two constraints are introduced to approximate the propagating waves to uniform plane waves. Firstly, the receiver must be located in the far field regions of the transmitter and the scatterers, if any. Secondly, the receiving waves are considered planar in the vicinity of the current receiver position, which is called the local planar zone of the current receiver location. The arriving waves are considered virtually emitted from an infinite source, whose first, or the reference, wave front is found at the transmitter location and the wave vectors point in the transmitter-receiver direction. Once the receiver moves out of the current local planar zone, the arriving wave vector direction changes accordingly to the new transmitter-receiver axis. The received signals at two locations are regarded as coming from the same uniform plane wave if a location is found in the local planar zone of the other location, i.e., if the angle between the two transmitter-receiver axes is small. Whenever the arriving waves at the receiver can be approximated as uniform plane waves, one can apply directly the kernel functions represented in arrival time $t_{o}$, received frequency $f_{o}$, receiver location $\mathbf{r}_{o}$ and receive wave vector $\mathbf{k}_{o}$ to characterize the transmission channel, seen from receivers.

\subsection{Application of the modeling framework at the transmitter side}

As mentioned above, the spatio-temporal modeling framework cannot be used to characterize the transmitted signals and the channels, as seen from the transmitters, since the space regions around the transmitters do not satisfy the plane wave approximation criteria. This also means that the multidimensional Fourier transform does not provide the duality between transmitter location $\mathbf{r}_{i}$ and transmit wave vector $\mathbf{k}_{i}$. The transmitted wave vector spectrum cannot be estimated from the transmitted signal in space domain, as was the case at the receiver side. In order to characterize fully systems and channels at transmitter side, the framework is modified by adding the AoD to the representation of kernel functions. The set of eight modified kernel functions, identified as $O(\cdot)$, and the input-output rela- 
Table 2 Ensemble of modified Kernel functions in space-time-wave vector-frequency

\begin{tabular}{lll}
\hline No. & Kernel function & Input-output relationship \\
\hline 1 & $O_{1}\left(\mathbf{r}_{\mathbf{o}}, t_{o} ; \mathbf{r}_{\mathbf{i}}, \psi_{i}, t_{i}\right)$ & $S_{o}\left(\mathbf{r}_{o}, t_{o}\right)=\iiint s_{i}\left(\mathbf{r}_{i}, \psi_{i}, t_{i}\right) O_{1}\left(\mathbf{r}_{o}, t_{o} ; \mathbf{r}_{i}, \psi_{i}, t_{i}\right) \mathrm{d} \mathbf{r}_{i} \mathrm{~d} \psi_{i} \mathrm{~d} t_{i}$ \\
2 & $O_{2}\left(\mathbf{r}_{\mathbf{o}}, t_{o} ; \mathbf{r}_{\mathbf{i}}, \psi_{i}, f_{i}\right)$ & $S_{o}\left(\mathbf{r}_{o}, t_{o}\right)=\iiint S_{i}\left(\mathbf{r}_{i}, \psi_{i}, f_{i}\right) O_{2}\left(\mathbf{r}_{o}, t_{o} ; \mathbf{r}_{i}, \psi_{i}, f_{i}\right) \mathrm{d} \mathbf{r}_{i} \mathrm{~d} \psi_{i} \mathrm{~d} f_{i}$ \\
3 & $O_{3}\left(\mathbf{r}_{\mathbf{o}}, f_{o} ; \mathbf{r}_{\mathbf{i}}, \psi_{i}, f_{i}\right)$ & $S_{o}\left(\mathbf{r}_{o}, f_{o}\right)=\iiint S_{i}\left(\mathbf{r}_{i}, \psi_{i}, f_{i}\right) O_{3}\left(\mathbf{r}_{o}, f_{o} ; \mathbf{r}_{i}, \psi_{i}, f_{i}\right) \mathrm{d} \mathbf{r}_{i} \mathrm{~d} \psi_{i} \mathrm{~d} f_{i}$ \\
4 & $O_{4}\left(\mathbf{r}_{\mathbf{o}}, f_{o} ; \mathbf{r}_{\mathbf{i}}, \psi_{i}, t_{i}\right)$ & $S_{o}\left(\mathbf{r}_{o}, f_{o}\right)=\iiint s_{i}\left(\mathbf{r}_{i}, \psi_{i}, t_{i}\right) O_{4}\left(\mathbf{r}_{o}, f_{o} ; \mathbf{r}_{i}, \psi_{i}, t_{i}\right) \mathrm{d} \mathbf{r}_{i} \mathrm{~d} \psi_{i} \mathrm{~d} t_{i}$ \\
5 & $O_{5}\left(\mathbf{k}_{\mathbf{o}}, t_{o} ; \mathbf{r}_{\mathbf{i}}, \psi_{i}, t_{i}\right)$ & $S_{o}\left(\mathbf{k}_{o}, t_{o}\right)=\iiint s_{i}\left(\mathbf{r}_{i}, \psi_{i}, t_{i}\right) O_{5}\left(\mathbf{k}_{o}, t_{o} ; \mathbf{r}_{i}, \psi_{i}, t_{i}\right) \mathrm{d} \mathbf{r}_{i} \mathrm{~d} \psi_{i} \mathrm{~d} t_{i}$ \\
6 & $O_{6}\left(\mathbf{k}_{\mathbf{o}}, t_{o} ; \mathbf{r}_{\mathbf{i}}, \psi_{i}, f_{i}\right)$ & $S_{o}\left(\mathbf{k}_{o}, t_{o}\right)=\iiint S_{i}\left(\mathbf{r}_{i}, \psi_{i}, f_{i}\right) O_{6}\left(\mathbf{k}_{o}, t_{o} ; \mathbf{r}_{i}, \psi_{i}, f_{i}\right) \mathrm{d} \mathbf{r}_{i} \mathrm{~d} \psi_{i} \mathrm{~d} f_{i}$ \\
7 & $O_{7}\left(\mathbf{k}_{\mathbf{o}}, f_{o} ; \mathbf{r}_{\mathbf{i}}, \psi_{i}, f_{i}\right)$ & $S_{o}\left(\mathbf{k}_{o}, f_{o}\right)=\iiint S_{i}\left(\mathbf{r}_{i}, \psi_{i}, f_{i}\right) O_{7}\left(\mathbf{k}_{o}, f_{o} ; \mathbf{r}_{i}, \psi_{i}, f_{i}\right) \mathrm{d} \mathbf{r}_{i} \mathrm{~d} \psi_{i} \mathrm{~d} f_{i}$ \\
8 & $O_{8}\left(\mathbf{k}_{\mathbf{o}}, f_{o} ; \mathbf{r}_{\mathbf{i}}, \psi_{i}, t_{i}\right)$ & $S_{o}\left(\mathbf{k}_{o}, f_{o}\right)=\iiint s_{i}\left(\mathbf{r}_{i}, \psi_{i}, t_{i}\right) O_{8}\left(\mathbf{k}_{o}, f_{o} ; \mathbf{r}_{i}, \psi_{i}, t_{i}\right) \mathrm{d} \mathbf{r}_{i} \mathrm{~d} \psi_{i} \mathrm{~d} t_{i}$ \\
\hline
\end{tabular}

tionships based on these functions are listed in Table 2 . The transmitted signal representation is also modified accordingly.

One observes that, at the transmitter side, the Fourier transform applies only on kernel functions with respect to transmit time variable $\left(t_{i}\right)$ to provide the kernel functions in frequency domain, i.e., $f_{i}$. Spatial variable $\mathbf{r}_{i}$ represents the transmitter location and it is independent from other variables, i.e., AoD $\psi_{i}$ and transmit time $t_{i}$. Variable $\psi_{i}$ represents the angle of departure or the transmit direction from the transmit antenna. In two-dimensional space, $\psi_{i}$ is the azimuth angle, whereas in three-dimensional space, $\psi_{i}$ is a pair of azimuth and elevation angles. $t_{i}$ is the time variable at transmitter. Marginalization integral can be performed to remove some of the dependent variables. For instance, using marginalization integrals on kernel functions with respect to AoD variable, $\psi_{i}$, leads to the kernel function presentation in space and time domains, or in space and frequency domains, at transmitter side.

The relationships between the set of kernel functions $O(\cdot)$ is depicted in Fig. 3. The kernel function relationship cube is built similarly to that in Fig. 2. Kernel func-

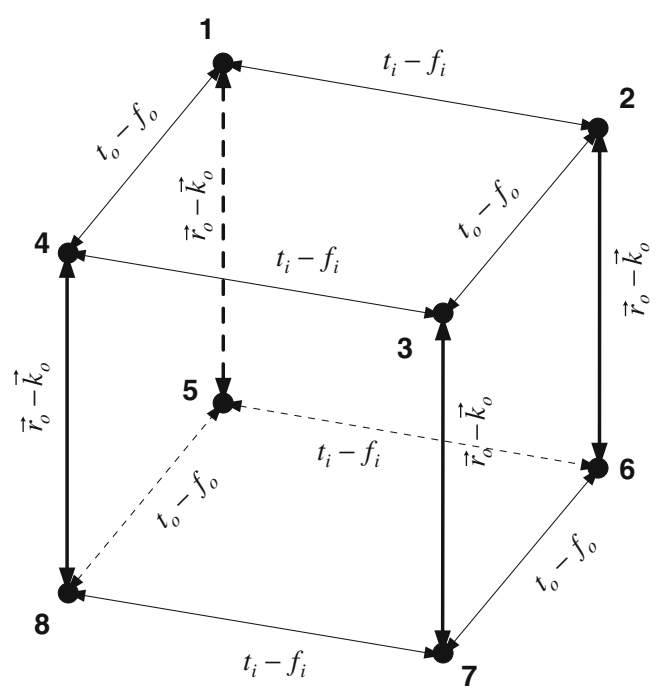

Fig. 3 Reduced kernel function relationship tions $O_{1}(\cdot), O_{2}(\cdot), O_{3}(\cdot)$ and $O_{4}(\cdot)$ are interconnected by Fourier transform on time and frequency variable pairs $t_{i}-f_{i}$ and $t_{o}-f_{o}$. Kernel functions $O_{5}(\cdot), O_{6}(\cdot)$, $\mathrm{O}_{7}(\cdot)$ and $\mathrm{O}_{8}(\cdot)$ are also interconnected by Fourier transform on time and frequency variable pairs $t_{i}-f_{i}$ and $t_{o}-f_{o}$. Kernel function pairs $O_{1}(\cdot)-O_{5}(\cdot), O_{2}(\cdot)-$ $\mathrm{O}_{6}(\cdot), \mathrm{O}_{3}(\cdot)-\mathrm{O}_{7}(\cdot)$ and $\mathrm{O}_{4}(\cdot)-\mathrm{O}_{8}(\cdot)$ are interconnected by three-dimensional Fourier transform on variable pair $\mathbf{r}_{o}-\mathbf{k}_{o}$.

\section{MIMO antenna array design}

In this section, we discuss the application of the reduced kernel functions, $O(\cdot)$, in order to design the transmit and receive antenna arrays for MIMO communications systems. Without loss of generality, we consider uniform linear array (ULA) antennas with $N_{T}$ elements at the transmitter and $N_{R}$ antenna elements at the receiver. The extension to other types of antenna arrays is straightforward. To maximize the channel capacity of a MIMO communications system, the sub-channels needs to be uncorrelated and the channel matrix needs to have high rank.

\subsection{Transmit antenna array design}

\subsubsection{Antenna element separation}

To determine the transmit antenna element separation, the transmit coherence spatial vector has to be estimated. For ULA antenna, the coherence spatial vector becomes the coherence distance. The estimation is based on the kernel correlation functions. The correlation function of kernel function $O_{1}(\cdot)$ is presented in Eq. 32:

$$
\begin{aligned}
& R_{O_{1} O_{1}}\left(\mathbf{r}_{o, 1}, \mathbf{r}_{o, 2}, t_{o, 1}, t_{o, 2} ; \mathbf{r}_{i, 1}, \mathbf{r}_{i, 2}, \psi_{i, 1}, \psi_{i, 2}, t_{i, 1}, t_{i, 2}\right) \\
& =E\left\{O_{1}\left(\mathbf{r}_{o, 1}, t_{o, 1} ; \mathbf{r}_{i, 1}, \psi_{i, 1}, t_{i, 1}\right) O_{1}^{*}\left(\mathbf{r}_{o, 2}, t_{o, 2} ; \mathbf{r}_{i, 2}, \psi_{i, 2}, t_{i, 2}\right)\right\}
\end{aligned}
$$

where $E\{\cdot\}$ is the ensemble average and ${ }^{*}$ is the conjugate operator. If the channel is wide sense stationary (WSS) in space, AoD and time domains, the correlation function becomes Eq. 33 . 
Marginalization integrals can be performed on the kernel correlation functions with respect to some variables in order to obtain compact kernel correlation functions [33]. Without loss of generality, $R_{O O}\left(\Delta \mathbf{r}_{i}, \Delta \psi_{i}, \Delta t_{i}\right)$ denotes the transmit correlation function of a WSS channel, as it can be estimated from any kernel function $O(\cdot)$ that contains variables $\mathbf{r}_{i}, \psi_{i}$, and $t_{i}$. The transmit coherence spatial vector, $\mathbf{r}_{i, c}$, or the transmit coherence distance in the case of ULA antenna, $d_{i, c}$, is the distance over which the transmit correlation function $R_{O O}\left(\Delta \mathbf{r}_{i}, \Delta \psi_{i}, \Delta t_{i}\right)$ drops below a given threshold, e.g., $50 \%$ of the maximum correlation peak. The transmit antenna elements must be separated by a distance greater than the transmit coherence distance $d_{i, c}$ in order to obtain sufficient sub-channel decorrelation.

$$
\begin{aligned}
R_{O_{1} O_{1}} & \left(\Delta \mathbf{r}_{o}, \Delta t_{o} ; \Delta \mathbf{r}_{i}, \Delta \psi_{i}, \Delta t_{i}\right) \\
=E\{ & O_{1}\left(\mathbf{r}_{o}, t_{o} ; \mathbf{r}_{i}, \psi_{i}, t_{i}\right) \\
\cdot & \left.O_{1}^{*}\left(\mathbf{r}_{o}-\Delta \mathbf{r}_{o}, t_{o}-\Delta t_{o} ; \mathbf{r}_{i}-\Delta \mathbf{r}_{i}, \psi_{i}-\Delta \psi_{i}, t_{i}-\Delta t_{i}\right)\right\}
\end{aligned}
$$

$$
\begin{aligned}
& R_{O_{5} O_{5}}\left(\Delta \mathbf{k}_{o}, \Delta t_{o} ; \Delta \mathbf{r}_{i}, \Delta \psi_{i}, \Delta t_{i}\right) \\
&=E\left\{O_{5}\left(\mathbf{k}_{o}, t_{o} ; \mathbf{r}_{i}, \psi_{i}, t_{i}\right)\right. \\
&\left.\cdot O_{5}^{*}\left(\mathbf{k}_{o}-\Delta \mathbf{k}_{o}, t_{o}-\Delta t_{o} ; \mathbf{r}_{i}-\Delta \mathbf{r}_{i}, \psi_{i}-\Delta \psi_{i}, t_{i}-\Delta t_{i}\right)\right\}
\end{aligned}
$$

$$
\begin{aligned}
R_{O O} & \left(\Delta \mathbf{k}_{o}, \Delta t_{o}\right) \\
= & \iiint R_{O_{5} O_{5}}\left(\Delta \mathbf{k}_{o}, \Delta t_{o} ; \Delta \mathbf{r}_{i}, \Delta \psi_{i}, \Delta t_{i}\right) \\
& \cdot \mathrm{d} \Delta \mathbf{r}_{i} \mathrm{~d} \Delta \psi_{i} \mathrm{~d} \Delta t_{i}
\end{aligned}
$$

$$
R_{O O}\left(\Delta \mathbf{k}_{o}\right)=\int R_{O O}\left(\Delta \mathbf{k}_{o}, \Delta t_{o}\right) \mathrm{d} \Delta t_{o}
$$

\subsubsection{Antenna element beam separation}

To further decrease the sub-channel correlation, directional antenna elements can be used. In this scenario, each transmit antenna element emits signal in a given direction. The element beams are separated also by an angle which is larger than the coherence angle of departure, $\psi_{i, c}$, defined as the angle over which the transmit correlation function, $R_{O O}\left(\Delta \mathbf{r}_{i}, \Delta \psi_{i}, \Delta t_{i}\right)$, is below a specified threshold.
Transmit correlation function $R_{O O}\left(\mathbf{r}_{i, m}, \mathbf{r}_{i, n}, \Delta \psi_{i}\right.$, $\Delta t_{i}$ ) represents the kernel correlation function between the $m$ th and $n$th transmit antenna elements. The coherence angle of departure estimated by this correlation function, $\psi_{i, c}(m, n)$, depends on these antenna elements. Taking into account the AoD coherence in designing the transmit beam of antenna elements further reduces the sub-channel correlation.

Functions $R_{O O}\left(\Delta \mathbf{r}_{i}, \Delta \psi_{i}, t\right)$ and $R_{O O}\left(\mathbf{r}_{i, m}, \mathbf{r}_{i, n}, \Delta \psi_{i}, t\right)$ are estimated at time instant $t$. This implies that the coherence angles of departure estimated from these functions are time-varying. A transmitter using these coherence angles, $\psi_{i, c}(t)$ and $\psi_{i, c}(m, n, t)$, in order to adaptively orient its beams obtains stable performance in fast time-varying channels.

\subsubsection{Spatial-frequency multiplexing}

Transmit correlation functions $R_{O O}\left(\Delta \mathbf{r}_{i}, \Delta \psi_{i}, \Delta f_{i}\right)$ describe the transmit correlation in space, in terms of angle of departure and frequency separation. The coherence frequency estimated from correlation function $R_{O O}\left(\Delta \mathbf{r}_{i}, \Delta \psi_{i}, \Delta f_{i}\right)$, i.e., $f_{i, c}$, represents the minimum frequency separation of transmit antenna elements to reduce the sub-channel correlation.

Similarly to coherence angle of departure, the coherence frequency $f_{i, c}(m, n)$, estimated from transmit correlation function $R_{O O}\left(\mathbf{r}_{i, m}, \mathbf{r}_{i, n}, \Delta f_{i}\right)$, represents the minimum frequency separation that the $m$ th and $n$th transmit antenna elements needed to ensure uncorrelated sub-channels. More specifically, the coherence frequency $f_{i, c}\left(m, n, \psi_{i, m}, \psi_{i, n}\right)$, estimated from transmit correlation function $R_{O O}\left(\mathbf{r}_{i, m}, \mathbf{r}_{i, n}, \psi_{i, m}, \psi_{i, n}, \Delta f_{i}\right)$, reflects the minimum frequency separation that the $m$ th and the $n$th antenna elements must follow when their beams orient in directions $\psi_{i, m}$ and $\psi_{i, n}$, respectively. A system built based on these coherence values is capable of performing spatial-frequency multiplexing.

\subsection{Receive antenna array design}

The application of the proposed framework in designing receive antenna arrays is also based on the second order statistics of the kernel functions, as was the case for the transmit antenna array design discussed in Section 8.1. The generally receive correlation function of a space and time WSS channel is:

$$
\begin{aligned}
& R_{O O}\left(\Delta \mathbf{r}_{o}, \Delta t_{o}\right)= \\
& \iiint R_{O_{1} O_{1}}\left(\Delta \mathbf{r}_{o}, \Delta t_{o} ; \Delta \mathbf{r}_{i}, \Delta \psi_{i}, \Delta t_{i}\right) \mathrm{d} \Delta \mathbf{r}_{i} \mathrm{~d} \Delta \psi_{i} \mathrm{~d} \Delta t_{i}
\end{aligned}
$$

Performing marginalization over $\Delta t_{i}$ variable, results in the receive correlation function is space, $R_{O O}\left(\Delta \mathbf{r}_{o}\right)$. The receive coherence distance $\left(d_{o, c}\right)$, estimated from 
the receive correlation function in space $\left(R_{O O}\left(\Delta \mathbf{r}_{O}\right)\right)$ represent the minimum antenna element separation in the receive antenna array.

The receive correlation functions, $R_{O_{5} O_{5}}(\cdot)$ and $R_{O O}(\cdot)$, in wave vector domain are presented in Eqs. 34-36. Differently from the situation observed at the transmitter side, the coherence receive wave vector, $\Delta \mathbf{k}_{o, c}$, contains both the minimum angle of arrival separation and receive frequency separation that the receive antenna elements needs to follow in order to guarantee low sub-channel correlation. This is because of the assumption made that the arrival waves are considered uniform plane waves.

\section{Illustrative examples: AoA estimation}

In this section, we use some field test data to illustrate the utilization of the practical modeling framework in estimating the angle of arrival. In our measurements, the sounding signal is the ATSC DTV signal. The transmitter with one transmit antenna is mounted on top of a television tower. The receiver with two receive antennas, separated by $2.1 \mathrm{~m}$ or $5.5 \lambda$ (wavelengths), is installed in a mobile laboratory unit. The spatial kernel functions, ${ }^{3} O\left(\mathbf{r}_{o} ; \mathbf{r}_{i}\right)_{\mid \mathbf{r}_{i}=\text { constant }}$, were estimated by using the pseudorandom number sequence PN511, embedded in the ATSC signal (part 2 in [34]), and a sliding correlator.

\subsection{AoA estimation at rural site}

The measurement site of this example is shown in Fig. 4. In order to perform spatial sampling, a mobile receiver with two receive antennas is moving at a speed of $60 \mathrm{~km} / \mathrm{h}$ from point 1 to point 2 and then from point 2 to point 1 . The antenna separation is $2.1 \mathrm{~m}$, or $5.5 \lambda$, at a carrier frequency of $788 \mathrm{MHz}$, and the sampling interval is $0.4 \mathrm{~m}$. The spatial kernel function estimated on the straight segment between the intersection and point 2 is used to estimate the angle of arrival. We assume that the channel is sufficiently stable during the measurement such that the samples are aligned in space within reasonable limits. Axis $x$ points from the intersection to point 2 , while axis $y$ is perpendicular to axis $x$ and points to the upper of Fig. 4.

\footnotetext{
${ }^{3}$ In these examples, we omit time and frequency variables. $O\left(\mathbf{r}_{o} ; \mathbf{r}_{i}\right)$ denotes the kernel function in transmit and receive location vectors while $O\left(\mathbf{k}_{o} ; \mathbf{r}_{i}\right)$ denote the kernel function in transmit location vector and receive wave vector.
}

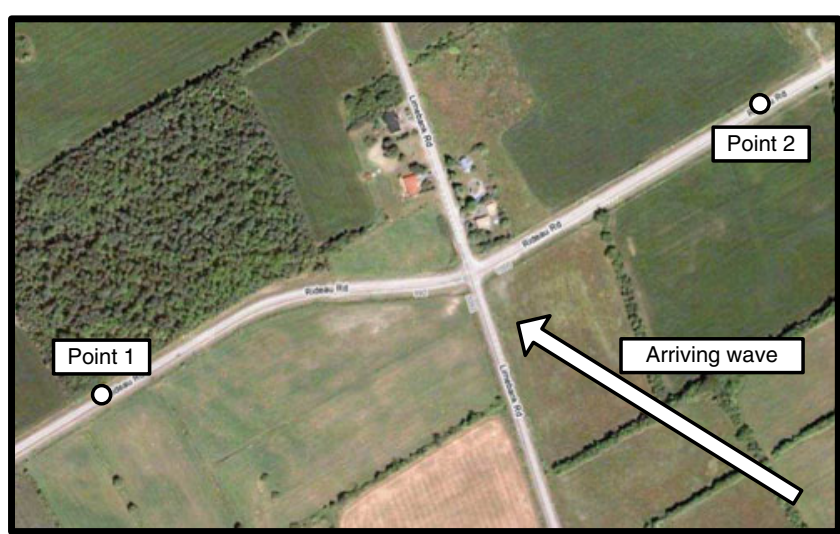

Fig. 4 Satellite photo of downlink rural measurement sites

Figure 5a shows the LOS components of the spatial kernel function, $O\left(\mathbf{r}_{o} ; \mathbf{r}_{i}\right)_{\mathbf{r}_{i}=\text { constant }}$, represented by a $4 \times 1,000$ matrix, for a downlink rural measurement. The sampling interval is $0.4 \mathrm{~m}$ along the $x$-axis and $2.1 \mathrm{~m}$

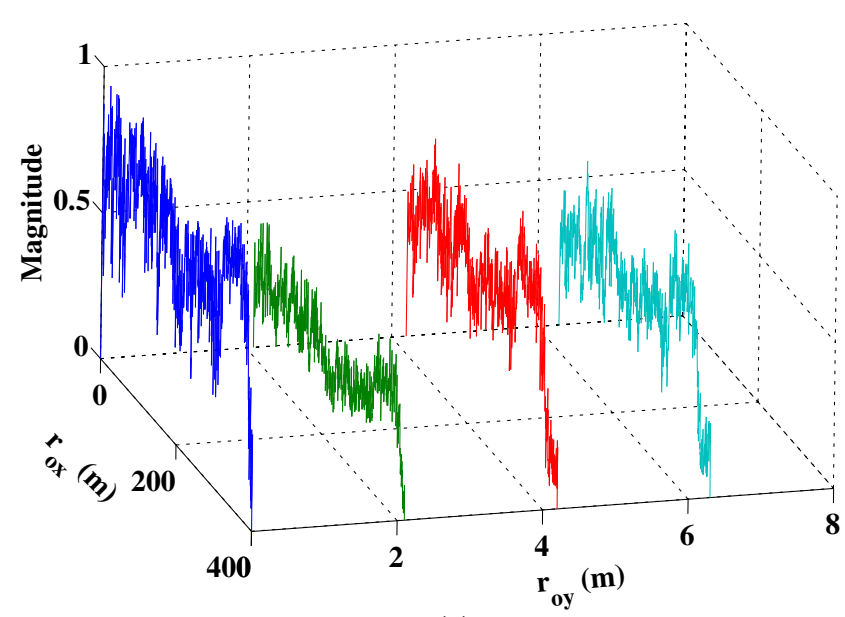

(a)

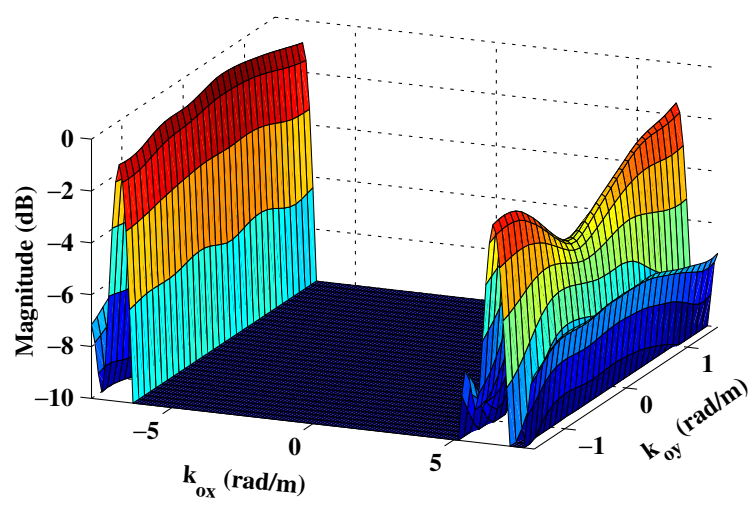

(b)

Fig. 5 Kernel functions (a) $\left.O\left(\mathbf{r}_{o}, \mathbf{r}_{i}\right)\right|_{\mathbf{r}_{i}=\text { constant }}$ and (b) $\left.O\left(\mathbf{k}_{o}, \mathbf{r}_{i}\right)\right|_{\mathbf{r}_{i}=\text { constant }}$ for downlink measurement at rural site 
along the $y$-axis. Applying a two-dimensional FFT on kernel function $O\left(\mathbf{r}_{\mathbf{o}}, \mathbf{r}_{\mathbf{i}}\right)_{\mid \mathbf{r}_{i}=\text { constant }}$, with respect to the receiver location vector, $\mathbf{r}_{o}$, gives us its dual kernel function, $O\left(\mathbf{k}_{\mathbf{0}}, \mathbf{r}_{\mathbf{i}}\right)_{\mid \mathbf{r}_{i}=\text { constant }}$.

Figure $5 \mathrm{~b}$ shows the resulting $2 \mathrm{D}$ wave vector spectrum. In this figure, the principal, or main, spectrum component is found at wave vector component $k_{o x}$ between -8 and $-7 \mathrm{rad} / \mathrm{m}$. There are two other strong spectrum components found at $k_{o x} \approx 6.5 \mathrm{rad} / \mathrm{m}, k_{o y} \approx$ $-1 \mathrm{rad} / \mathrm{m}$, and at $k_{o x} \approx 6.5 \mathrm{rad} / \mathrm{m}, k_{o y} \approx 1 \mathrm{rad} / \mathrm{m}$. Note that for our measurements, the spatial resolution in the $y$-axis is limited since only four sampling points at a sampling step of $2.1 \mathrm{~m}$ are collected in the $y$ direction at each sampling instant. Therefore, the information along the $k_{y}$-axis in the wave vector domain is not as precise as for the wave vector component along the $k_{x}$-axis. For this measurement, the carrier frequency is $788.31 \mathrm{MHz}$, the AoA of the LOS component is $120^{\circ}$. Therefore, the $k_{x}$ component of the LOS component is $-8.25 \mathrm{rad} / \mathrm{m}$, which is very close to the value estimated from the kernel function $O\left(\mathbf{k}_{o}, \mathbf{r}_{i}\right)_{\mid \mathbf{r}_{i}=\text { constant }}$. The spectrum component found at $k_{o x}=-8 \sim-7 \mathrm{rad} / \mathrm{m}$ corresponds to the LOS component, while the components found at $k_{o x} \approx 6.5 \mathrm{rad} / \mathrm{m}$ are the reflected components.

\subsection{AoA estimation at suburban site}

The measurement site of this example is depicted in Fig. 6. Similarly to the rural site measurement, the mobile unit is traveling from point 1 to point 2 and then from point 2 to point 1 to perform the spatial sampling process. Kernel function $O\left(\mathbf{r}_{o} ; \mathbf{r}_{i}\right)_{\left.\right|_{i}=\text { constant }}$, estimated on the straight segment from point 1 to the intersection, is used in this example. Axis $x$ point from point 1 to the intersection. Axis $y$ is perpendicular to axis $x$ and point to the lower right corner of Fig. 6 . The speed of

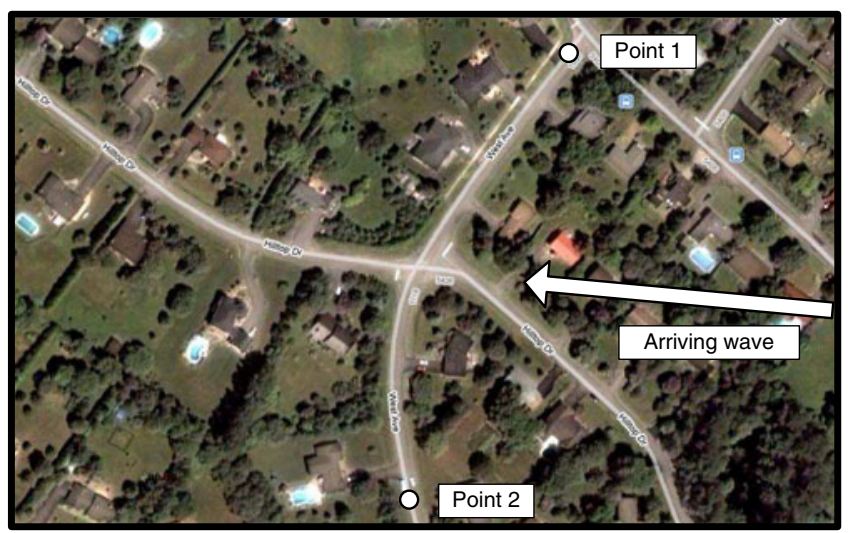

Fig. 6 Satellite photo of downlink suburban measurement site the mobile unit is $30 \mathrm{~km} / \mathrm{h}$. The spatial sampling in axis $x$ is $0.2 \mathrm{~m}$ and in axis $y$ is $2.1 \mathrm{~m}$.

Figure $7 \mathrm{a}$ shows the LOS components of spatial kernel function $O\left(\mathbf{r}_{\mathbf{o}}, \mathbf{r}_{\mathbf{i}}\right)_{\mid \mathbf{r}_{i}=\text { constant }}$, while Fig. 7b shows the dual kernel function $O\left(\mathbf{k}_{\mathbf{0}}, \mathbf{r}_{\mathbf{i}}\right)_{\mid \mathbf{r}_{i}=\text { constant }}$. One observes clearly that there are three spectrum components found at $\mathbf{k}_{o_{1}}=\left(k_{o_{1} x}, k_{o_{1} y}\right) \approx(11,-1.2) \mathrm{rad} / \mathrm{m}$, at $\mathbf{k}_{O_{2}}=\left(k_{o_{2} x}, k_{o_{2} y}\right) \approx(11,-0.1) \mathrm{rad} / \mathrm{m}$ and at $\mathbf{k}_{o_{3}}=$ $\left(k_{o_{3} x}, k_{o_{3} y}\right) \approx(11,1) \mathrm{rad} / \mathrm{m}$. For this example, the carrier frequency is $788.31 \mathrm{MHz}$, the AoA of the LOS component is $50^{\circ}$. Therefore, the $k_{x}$ component of the LOS component is $10.6 \mathrm{rad} / \mathrm{m}$. This is very close to the values extracted from the received wave vector spectrum, $O\left(\mathbf{k}_{\mathbf{0}}, \mathbf{r}_{\mathbf{i}}\right)_{\mid \mathbf{r}_{i}=\text { constant }}$, mentioned above. The three wave vector spectrum components found in Fig. $7 \mathrm{~b}$ at positive $k_{o x}$ correspond to the LOS component. At negative values of $k_{o x}$, we find some low-magnitude spectrum components, which likely correspond to the reflected waves.

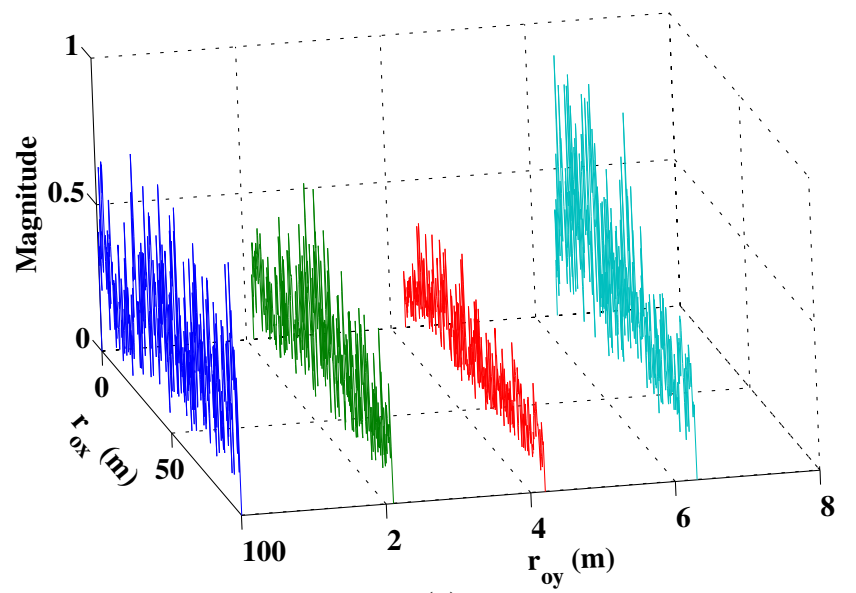

(a)

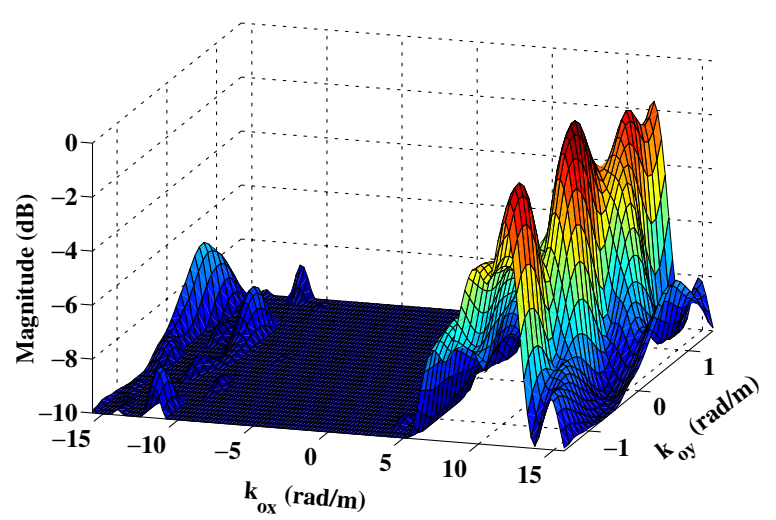

(b)

Fig. 7 Kernel functions (a) $\left.O\left(\mathbf{r}_{o}, \mathbf{r}_{i}\right)\right|_{\mathbf{r}_{i}=\text { constant }}$ and (b) $\left.O\left(\mathbf{k}_{o}, \mathbf{r}_{i}\right)\right|_{\mathbf{r}_{i}=\text { constant }}$ for downlink measurement at suburban site 


\section{Conclusions}

In this paper, a new theoretical framework is proposed for MIMO channel characterization and modeling. The proposed spatio-temporal modeling framework allows us to represent MIMO channels and systems in space, time, wave vector, and frequency domains. The relationships between space and time and between wave vector and frequency are explicitly described. The proposed theoretical framework consists of sixteen kernel functions. The kernel functions are interconnected by Fourier transforms. Since the theoretical framework is based on uniform plane wave propagation, the application of the spatio-temporal framework to realistic propagation situations is not straightforward. For this reason, a practical framework is proposed to approximate the theoretical framework in realistic scenarios. The practical framework consists of eight kernel functions, which represent channels in space, time, receive wave vector, frequency, and direction of departure. Time-frequency duality exists at both the receiver and the transmitter sides, whereas the wave vector-location vector duality exists only at the receiver side. Applications of the practical framework in designing transmit and receive antenna arrays and in estimating angle of arrival using field test measurement data are presented.

Acknowledgements The authors are greatly indebted to $\mathrm{Mr}$. Sébastien Laflèche, Mr. Robert Gagnon, Mr. Benoit Ledoux, Mr. Charles Nadeau, Mr. Gilles Gagnon, and Mr. Bernard Caron from the Television Broadcast Technologies Research Group at the Communication Research Center in Ottawa, who very kindly provided the mobile channel measurement facilities essential to the field experiments conducted for the data analysis presented in this paper.

Open Access This article is distributed under the terms of the Creative Commons Attribution Noncommercial License which permits any noncommercial use, distribution, and reproduction in any medium, provided the original author(s) and source are credited.

\section{References}

1. Ghosh A, Ratasuk R, Mondal B, Mangalvedhe N, Thomas $\mathrm{T}$ (2010) LTE-advanced: next-generation wireless broadband technology [Invited Paper]. IEEE Wirel Commun Mag 17(3):10-22

2. Ahmadi S (2009) An overview of next-generation mobile WiMAX technology. IEEE Commun Mag 47(6):84-98

3. Foschini GJ (1996) Layered space-time architecture for wireless communication in a fading environment when using multi-element antennas. Bell Labs Tech J 1(2):41-59

4. Alamouti SM (1998) A Simple Transmit Diversity Technique for Wireless Communications. IEEE J Sel Areas Commun 16(8):10
5. Paulraj A, Nabar R, Gore D (2003) Introduction to spacetime wireless communications. Cambridge University Press, Cambridge

6. Sterian CED, Wu Y, Pätzold M (2008) Superquasiorthogonal space-time trellis codes for four transmit antennas with rectangular signal constellations. Ann Telecommun 63(5-6):331-350

7. Bouvet P-J, Hélard M, Nir VL (2006) Simple iterative receivers for MIMO LP-OFDM systems. Ann Telecommun 61(5-6):578-601

8. Naguib A, Paulraj A, Kailath T (1994) Capacity improvement with base-station antenna arrays in cellular CDMA. IEEE Trans Veh Technol 43(3):691-698

9. Molisch A, Win M, Choi Y, Winters J (2005) Capacity of MIMO systems with antenna selection. IEEE Trans Wirel Commun 4(4):1759-1772

10. Winters J (1987) On the capacity of radio communication systems with diversity in a Rayleigh fading environment. IEEE J Sel Areas Commun 5(5):871-878

11. Winters J, Salz J, Gitlin R (1994) The impact of antenna diversity on the capacity of wireless communication systems. IEEE Trans Commun 42(2):1740-1751

12. Foschini G, Gans M (1998) On limits of wireless communications in a fading environment when using multiple antennas. Wirel Pers Commun 6(3):311-335

13. Shiu D, Foschini G, Gans M, Kahn J et al (2000) Fading correlation and its effect on the capacity of multielement antenna systems. IEEE Trans Commun 48(3):502-513

14. Chuah C, Tse D, Kahn J, Valenzuela R (2002) Capacity scaling in MIMO wireless systems under correlated fading. IEEE Trans Inf Theory 48(3):637-650

15. Chizhik D, Foschini GJ, Gans MJ, Valenzuela RA (2002) Keyholes, correlations, and capacities of multielement transmit and receive antennas. IEEE Trans Wirel Commun 1(2):361-368

16. Gesbert D, Bolcskei H, Gore DA, Paulraj AJ (2002) Outdoor MIMO wireless channels: models and performance prediction IEEE Trans Commun 50(12):1926-1934

17. Almers P, Tufvesson F, Molisch AF (2003) Measurement of keyhole effect in a wireless multiple-input multiple-output (MIMO) channel. IEEE Commun Lett 7(8):373-375

18. Sayeed A, Sendonaris A, Aazhang B (1998) Multiuser detection in fast-fading multipath environments. IEEE J Sel Areas Commun 16(9):1691-1701

19. Bhashyam S, Sayeed AM, Aazhang B (2000) Time-selective signaling and reception for communication over multipath fading channels. IEEE Trans Commun 48(1):83-94

20. Onggosanusi E, Sayeed A, Van Veen B (2000) Canonical space-time processing for wireless communications. IEEE Trans Commun 48(10):1669-1680

21. Durgin GD (2003) Space-time wireless channels. Prentice Hall

22. Molisch A, Asplund H, Heddergott R, Steinbauer M, Zwick $\mathrm{T}$ (2006) The COST259 directional channel model-part I: overview and methodology. IEEE Trans Wirel Commun 5(12):3421-3433

23. Asplund H, Glazunov A, Molisch A, Pedersen K, Steinbauer M (2006) The COST 259 directional channel model-part II: macrocells. IEEE Trans Wirel Commun 5(12):3434-3450

24. Guguen P, Zein GE (2004) Les techniques multi-antennes pour les Réseaux Sans Fil (in French). Hermes Science, Paris

25. Chandran S, Guguen P, Zein GE (editors) (2004) Adaptive antenna arrays, trends and applications. Springer, Berlin. pp 360-376 
26. Sayeed A (2002) Deconstructing multiantenna fading channels. IEEE Trans Signal Process 50(10):2563-2579

27. Fluerasu A, Letrou C (2009) Gaussian beam launching for 3D physical modeling of propagation channels. Anna Telecommun 64(11-12):763-776

28. Bello PA (1964) Time-frequency duality. IEEE Trans Inf Theory 10(1):18-33, 1

29. Zemanian AH (1965) Distribution theory and transform analysis: an introduction to generalized functions, with applications. McGraw-Hill, New York
30. Dearholt DW, McSpadden WR (1973) Electromagnetic wave propagation. McGraw-Hill, New York

31. Bello PA (1963) Characterization of randomly time-variant linear channels. IEEE Trans Commun Syst 11(4):360-393, 12

32. Balanis CA (2005) Antenna theory analysis and design, 3rd edn. Wiley, New York

33. Papoulis A, Pillai SU (2001) Probability, random variables and stochastic processes, 4th edn. McGraw-Hill, New York

34. A/53: ATSC Digital Television Standard, parts 1-6 advanced television systems committee 2007 